\title{
Effets du faisceau laser sur la fonction visuelle
}

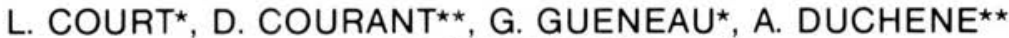

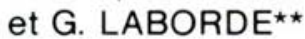

\begin{abstract}
RESUME
Les dangers présentés par l'emploi des lasers, en particulier les risques oculaires, ont imposé la définition de limites d'exposition. La détermination de cette valeur nécessite de connaître les parametres physiques et biologiques de l'exposition mais aussi de définir la notion de critère d'atteinte. La description de ces paramètres, des mécanismes impliqués dans les effets biologiques des lasers et de différents résultats expérimentaux illustre les difficultés rencontrées dans la détermination des limites d'exposition et l'incertitude des normes actuelles.
\end{abstract}

\section{ABSTRACT}

The risks due to the use of lasers, particularly ocular hazards, have brought the notion of exposure limits. The determination of this value requires both the knowledge of the physical and biological parameters of exposure and the definition of the concept of damage criteria. The parameters of the mechanisms involved in the biological effects of lasers and the various experimental results are described thus emphasizing the difficulties encountered in the determination of the exposure limits and the incertitude of the present guidelines.

Les dangers présentés par l'utilisation des lasers et ceux plus particulièrement liés au rayonnement sont dus essentiellement aux atteintes oculaires. Si la détermination des valeurs limites d'exposition impose une connaissance précise des paramètres physiques et des effets biologiques associés, l'influence d'un faisceau laser sur la fonction visuelle doit être évaluée dans une perspective dynamique de physiologie sensorielle. Le seuil doit exprimer la valeur limite d'une relation existant entre l'énergie ou la puissance dissipée au sein de la structure biologique exposée et l'apparition de la plus petite atteinte fonctionnelle ou lésion détectable susceptible d'entraîner un dommage. Cette définition introduit deux concepts : le choix du critère, atteinte réversible ou irréversible et la mesure de l'échange énergétique associé. II apparaît, ainsi, que les paramètres biologiques et physiques constituent un ensemble dont les

* Centre de recherche du service de santé des armées, 1 bis, rue du Lt Raoul Batany, F 92140 Clamart.

** Commissariat à l'énergie atomique, IPSN, Département de protection sanitaire, BP 6, 92260 Fontenay-aux-Roses. 
éléments réagissent les uns sur les autres dans une dynamique complexe. Leur description, la définition des critères, des mécanismes d'action et l'évocation de résultats expérimentaux illustreront les difficultés rencontrées dans l'analyse de l'atteinte fonctionnelle et dans la détermination des limites d'exposition et permettront d'évoquer les incertitudes des normes actuelles.

\section{LES PARAMETRES PHYSIQUES D'UNE EXPOSITION}

Ils varient avec le type de laser utilisé - continu ou à impulsions accordé sur une fréquence connue ou accordable, c'est-à-dire à longueur d'onde d'émission variable.

Ce sont :

$1^{\circ}$ La source, ponctuelle ou plus large, et par là l'angle sous lequel est vu, de l'observateur, la source lumineuse. On sait que tous les lasers au faisceau collimaté sont assimilés à des sources ponctuelle, si l'angle $\alpha$ sous lequel est vu la source est inférieur à un angle $\alpha$ minimum ( $\alpha \mathrm{MIN})$; pour une valeur supérieure à $\alpha \mathrm{MIN}$, la source est dite "étendue". La valeur de cet angle $\alpha$ MIN est une fonction complexe précisée dans les normes citées en référence.

$2^{\circ}$ La nature du faisceau, sa divergence et son caractère direct, réfléchi ou diffusé.

$3^{\circ}$ La longueur d'onde :

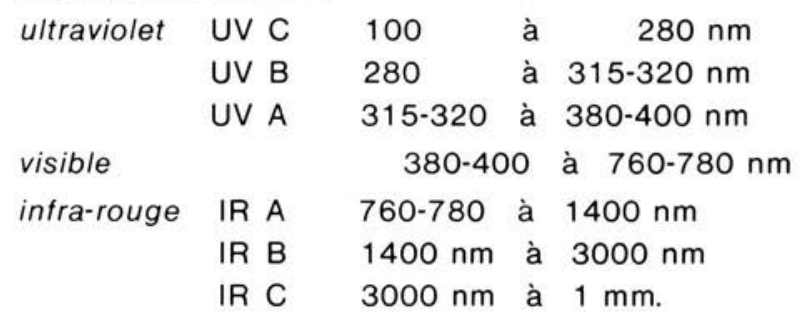

$4^{\circ}$ Le temps: la durée totale d'exposition, qu'elle soit instantanée ou chronique, que le laser soit continu ou à impulsion, mais aussi la durée de l'impulsion, sa fréquence, le temps séparant chaque impulsion dans le train d'impulsions lui-même, en d'autres termes la distribution temporelle de l'énergie.

5० Enfin, l'énergie et/ou la puissance absorbée par la structure exposée. L'expression est différente selon que le rayonnement est direct ou diffusé et, selon le mode de fonctionnement du laser, pulsé ou continu.

Le tableau I résume ces données. 
TABLEAU I

\section{Laser pulsé}

rayonnement direct

- en densité surfacique d'énergie (radiant exposure - $\mathrm{H}$ ) exprimée en joules par $\mathrm{cm}^{2}\left(\mathrm{~J} . \mathrm{cm}^{-2}\right)$

rayonnement diffusé visible et IR - A

- en densité surfacique d'énergie par unité d'angle solide, c'est-à-dire en joules par $\mathrm{cm}^{2}$ par steradian $\left(\mathrm{J} . \mathrm{cm}^{-2} \cdot \mathrm{sr}^{-1}\right)$

\section{Laser continu}

$>250 \mathrm{~ms}$
- en irradiance $E$, quotient du flux incident par la surface de l'élément irradié, exprimée en watts par $\mathrm{cm}^{2}\left(\mathrm{~W} . \mathrm{cm}^{-2}\right)$

- en radiance $L$ ou en unité de puissance par unité d'angle solide et par unité de surface, c'est-à-dire en watts par $\mathrm{cm}^{2}$ et par steradian $\left(\mathrm{W} . \mathrm{cm}^{-2} . \mathrm{sr}^{-1}\right)$

\section{LES PARAMETRES BIOLOGIQUES}

Le globe oculaire peut être assimilé chez l'homme à une sphère placée dans une cavité osseuse, l'orbite, ouverte en avant et protégée uniquement par le rideau palpébral et se déplaçant sous l'action de six muscles oculo-moteurs. Ce globe est composé de deux segments de sphère accolés et de structures intermédiaires.

$1^{\circ}$ Le segment antérieur transparent constitué par la cornée au contact de l'air ambiant par l'intermédiaire du film lacrymal en avant, de l'humeur aqueuse en arrière. Celle-ci remplit la chambre antérieure du globe délimité en arrière par le rideau irien au centre duquel se trouve la pupille. La cornée, épaisse de $1 \mathrm{~mm}$ environ, est constituée de 5 couches :

- un épithélium externe de faible épaisseur se reconstituant en $48 \mathrm{~h}$ lorsqu'il est lésé et s'appuyant sur :

- une membrane dite de Bowmann ;

- une couche épaisse constituée de fibres parallèles qui, lésées, guérissent par une cicatrice opaque ;

- une membrane élastique interne, membrane de Descemet qui ne se régénère pas et qui, lésée, laisse l'humeur aqueuse envahir les couches cornéennes centrales, provoquant un œdème entraînant une baisse de l'acuité visuelle ;

- un endothélium interne, couche monocellulaire au contact de l'humeur aqueuse.

Elle est transparente au rayonnement visible et au proche infra-rouge. Elle est sensible comme la conjonctive protégeant la face antérieure du globe et tapissant la face postérieure des paupières, aux infra-rouges lointains et aux ultra-violets.

$2^{\circ}$ Le segment postérieur composé de 3 couches :

- la sclérotique, enveloppe oculaire externe, opaque résistante et se continuant en avant par la cornée ; 
- la choroïde, épaisse et vasculaire au rôle nutritif, continuée en avant par l'iris, diaphragme musculaire au diamètre variable ;

- la rétine, la plus interne, élément sensoriel, constitué de dehors en dedans par l'épithélium pigmenté adhérent à la choroïde et protégeant les photorécepteurs, cônes et bâtonnets, les cellules bipolaires, puis les cellules ganglionnaires que prolongent les fibres nerveuses constituant le nerf optique. La topographie de la rétine, comme sa constitution, variable selon les espèces, se répartit en 3 zones chez l'homme :

- une zone aveugle ou papille, endroit d'émergence des fibres nerveuses sortant du globe pour constituer le nerf optique ;

- une zone périphérique plus riche en bâtonnets ;

- une zone centrale ou macula centrée sur la fovea peuplée de cônes. Cette zone située à $3 \mathrm{~mm}$ environ du bord temporal de la papille présente la sensibilité maximale; elle est le support de l'acuité visuelle et du sens chromatique, alors que la zone périphérique riche en bâtonnets est surtout utile en vision nocturne. L'acuité visuelle, maximale au centre de la macula n'atteint que le quart de sa valeur pour une excentricité de $5^{\circ}$ et $5 / 100$ à $20^{\circ}$.

\section{$3^{\circ}$ Les structures intermédiaires}

Elles se situent entre ces deux segments délimitant la chambre antérieure remplie d'humeur aqueuse sécrétée par le corps ciliaire et la chambre postérieure remplie de vitré ; ce sont l'iris et le cristallin.

- l'iris a une structure proche de la choroïde et possède des muscles dilatateurs et constricteurs réalisant l'ouverture de la pupille dont le diamètre varie de 2 à $3 \mathrm{~mm}$ en adaptation diurne à $7-8 \mathrm{~mm}$ en adapta-

- tion nocturne ;

- le cristallin, lentille transparente biconvexe située juste en arrière de l'iris et amarrée par des fibres contractiles au corps ciliaire et à l'iris. Cette lentille, au pouvoir d'accommodation diminuant avec l'âge, de $14 \mathrm{D}$ à 14 ans à $4 \mathrm{D}$ à 40 ans et $1 \mathrm{D}$ à 60 ans, focalise le faisceau lumineux sur la rétine.

Ce système oculaire constitue, toutefois, un système dynamique assurant dans une certaine mesure, de lui-même, une protection physiologique par:

- les paupières, aux mouvements spontanés et réflexes de fermeture, déclenchés en 150 à $250 \mathrm{~ms}$ par une illumination élevée (réflexe palpébral) ou plus rapidement mais de façon aléatoire à la suite d'un stimulus nociceptif sur la cornée c'est-à-dire provoquant un effet nocif sur l'architecture de la cornée ;

- le film lacrymal opaque aux infrarouges lointains et absorbant une partie de la chaleur par échauffement et évaporation ;

- la pupille, qui adaptant son diamètre au niveau d'éclairage ambiant, protège la rétine en ajustant le flux énergétique pénétrant dans l'œil ;

- les mouvements oculaires et en particulier le micronystagmus de fréquence variable de 5 à $10 \mathrm{cps}$;

- la vascularisation rétinienne très importante assurant une diffusion rapide du flux thermique. 
Ainsi, le globe oculaire sur le plan optique peut être assimilé à un système optique réduit au caractère dynamique réagissant au flux énergétique incident.

Aussi, les facteurs biologiques entrant en jeu dans les inductions des effets oculaires des lasers sont les suivants :

- Le diamètre pupillaire, fonction du niveau d'adaptation et qui varie de 2 à $7,5 \mathrm{~mm}$. Le flux lumineux entrant dans la pupille varie comme le carré du diamètre pupillaire. Le rapport pour une longueur d'onde donnée de l'irradiance rétinienne à l'irradiance de la cornée appelé gain optique ou magnification factor ou strehl ratio [12] varie de $10^{4}$ pour une pupille au diamètre voisin de $2 \mathrm{~mm}$, à $10^{5}$ pour un diamètre de $5 \mathrm{~mm}$ et à $2,5 \times 10^{5}$ pour un diamètre de $7,5 \mathrm{~mm}$.

- La formation d'image ou son absence : la dimension de l'image rétinienne est un facteur important [1]. On rappelle que compte tenu des aberrations du système optique oculaire, des micromouvements, l'image d'une source ponctuelle a une dimension minimale de l'ordre de 10-20 $\mu \mathrm{m}$.

- La localisation de l'image sur la rétine : une lésion à la périphérie est généralement beaucoup moins sévère qu'une atteinte fovéale entraînant, sinon la cécité, un scotome central important (lacune et/ou amputation du champ visuel).

- Le cœfficient d'absorption des milieux transparents, cornée, humeur aqueuse, vitrée, cristallin, épithélium pigmentaire, c'est-à-dire la structure du globe oculaire concernée et la nature et l'importance de la pigmentation [2, 3, 8]. L'importance de l'atteinte est fonction de l'énergie absorbée, de la surface stimulée, de sa localisation sur la rétine, de la transmittance des milieux oculaires et de la pigmentation variable selon la race.

Les lasers à impulsions et surtout ceux dont la fréquence de relaxation est élevée sont les plus dangereux. On admet qu'un laser est continu lorsque la durée d'émission est supérieure ou égale à $250 \mathrm{~ms}$, temps moyen du réflexe palpébral quand il existe. La protection qu'assure les réflexes photo et oculomoteurs est susceptible de jouer lors d'illumination continue; elle est sans effet lorsqu'il s'agit de lasers pulsés, aux impulsions courtes.

\section{LA NATURE DES ATTEINTES}

\section{III.1. Cornée}

Les atteintes cornéennes directes ou indirectes varient en fonction de la longueur d'onde. Comme au niveau de la conjonctive, l'illumination UV provoque en quelques heures une atteinte sévère. II apparaît une photokératite très douloureuse évoluant, en général, favorablement. Avec les IR et même les UV, il s'agit, avec des flux incidents faibles, d'une modification progressive de la courbure cornéenne puis, à un degré plus élevé, d'une opacité arrondie de diamètre apparent égal au faisceau. Cette opacité peut se creuser, se percer en son centre laissant sourdre l'humeur aqueuse alors que les berges opaques se réduisent progressivement. 
Les lésions indirectes sont à la fois des modifications de la morphologie de la cornée, mais aussi une dégénérescence plus ou moins superficielle, se surchargeant de dépôt calcique. Elle est, en général, la conséquence d'un œdème avec apparition de néo-vaisseaux.

Ainsi, quelques jours après l'illumination, des opacités grisâtres arrondies siégeant au-dessous de l'épithélium et dans les couches superficielles du stroma cornéen peuvent apparaître; elles sont centrales ou paracentrales et évoluent rapidement vers une zone totalement opaque. Ces lésions seraient secondaires à l'absorption de l'énergie au niveau de l'épithélium pigmenté de l'iris.

\section{III.2. Cristallin}

Ces lésions évoluent peu en fonction du temps. D'apparition plus ou moins rapide, elles ne disparaissent jamais et respectent en général la capsule antérieure. Elles sont le plus souvent de forme ovoïde au grand axe se situant dans la direction du faisceau incident, de coloration gris blanchâtre avec un maximum d'opacité au centre. Ceci traduit une altération définitive des protéines du cristallin.

\section{3. Iris}

La répétition d'impacts seule crée une altération se caractérisant par la migration des pigments dans la chambre antérieure dont l'humeur aqueuse réagit. Au niveau de l'impact, une zone de pigmentation anormale apparaît entourée d'une couronne en relief évoquant l'œdème local. Ces lésions cicatrisent et évoluent favorablement en trois semaines, si elles sont ponctuelles et rares. Larges et répétées, elles se traduisent par une paralysie de l'iris en myosis plus ou moins serré, une atrophie pouvant évoluer vers la déchirure plus ou moins complexe.

\section{III.4. Rétine}

- Lésions : en général arrondies, bien circonscrites, de dimension variable selon le diamètre de l'image, elles évoluent de la dépigmentation à l'hémorragie en goutte envahissant le vitré, pour les lésions majeures.

- Atteinte fonctionnelle : il existe entre la lésion rétinienne décelable à l'examen ophtalmoscopique et l'éblouissement physiologique, des atteintes fonctionnellles de la rétine se traduisant par un phototraumatisme que seule révèle une baisse de la sensibilité rétinienne dont l'adaptation à l'obscurité, comme le sens chromatique peuvent être définitivement modifiés. Ces phénomènes sont réversibles, irréversibles ou semi-réversibles et varient selon l'importance de l'image, mais apparaissent souvent plus importants lorsqu'il s'agit d'illumination continue en lumière diffusée à faible niveau. 


\section{MECANISMES GENERAUX DES EFFETS BIOLOGIQUES DES LASERS}

Le rayonnement laser se distingue de la plupart des autres types de rayonnements par la cohérence du faisceau. Le principal phénomène à l'origine de tous les types de dommages causés par le rayonnement laser est l'absorption de ce rayonnement par le système biologique. L'absorption est fonction de la distribution spatio-temporelle de l'énergie et elle constitue un processus spécifique de la longueur d'onde. C'est ce facteur qui détermine le tissu qu'un laser particulier est susceptible d'endommager.

Décrire les effets biologiques d'un faisceau laser, c'est décrire les modes d'action de la lumière sur les tissus vivants et dégager les principaux effets qui peuvent aider à comprendre l'action du laser sur le matériel biologique.

\section{IV.1. Les effets thermiques}

Ce sont les effets les plus importants, les mieux étudiés et les plus utilisés en biologie et en médecine. Ils sont caractéristiques de la région infrarouge mais s'étendent également à la partie visible du spectre. Lorsqu'un système a absorbé une énergie rayonnante suffisante, ses molécules constituantes éprouvent une vibration accrue, qui se traduit par une augmentation de la concentration thermique. Les molécules reviennent à leur état initial par une thermalisation de l'énergie absorbée, c'est-à-dire un dégagement de chaleur. Ainsi, la plupart des dommages créés par les lasers sont dus à l'échauffement du ou des tissus absorbants. Une élévation thermique de $10^{\circ} \mathrm{C}$ à $25^{\circ} \mathrm{C}$ suffit à provoquer localement une dénaturation des protéines et la mort cellulaire. A l'intérieur de la zone lésée, les cellules présentent des symptômes de brûlure. Si l'on chauffe encore plus, les tissus se rétractent par suite de l'évaporation d'une partie de leur contenu en eau, provoquant une nécrose de coagulation irréversible. Si l'on chauffe plus intensément encore, les tissus peuvent être volatilisés.

Après des études pratiques et théoriques, divers modèles ont été proposés pour tenter de prévoir l'étendue et la nature des lésions biologiques induites par les modifications thermiques [25, 29, 30].

Ces modèles sont, en général, confirmés par l'ensemble des résultats expérimentaux obtenus pour des durées d'exposition supérieures à la microseconde. Pour des durées plus faibles, d'autres phénomènes radicalement nouveaux viennent s'ajouter et le modèle thermique est alors insuffisant pour rendre compte des lésions observées.

\section{IV.2. Les effets photochimiques}

II existe d'autres types d'action de la lumière laser sur les tissus vivants que les effets thermiques précédemment décrits. Au premier rang viennent les effets photochimiques dont nous connaissons de nombreux exemples naturels: la synthèse chlorophyllienne, la formation de la vitamine $D$ dans l'organisme humain, le mécanisme de la vision. L'absorption, au lieu d'être globale, n'intéresse qu'une substance constituante 
de la cellule dont les molécules passent à un état excité. L'énergie d'excitation emmagasinée peut, au lieu de se dissiper en chaleur, provoquer des réactions chimiques in situ. Ce mode d'action, qui est caractéristique du domaine ultra-violet et du spectre visible, est souvent considéré comme responsable des lésions aux faibles niveaux d'exposition.

\section{IV.3. Les effets électromagnétiques}

Du fait de sa cohérence spatiale et lorsque l'on considère les puissances de crête considérables délivrées par certains types de laser, le champ électromagnétique lié au faisceau laser peut atteindre des valeurs considérables de l'ordre de $10^{7}$ à $10^{12} \mathrm{~V} \cdot \mathrm{m}^{-2}$. On conçoit donc aisément que des tirs lasers puissants puissent détruire toute organisation moléculaire et entraîner des ruptures de liaison. A un degré moindre, il semble que le rayonnement laser soit capable de modifier la conductivité et la constante diélectrique des tissus, ainsi que la polarisation membranaire des cellules entraînant des désordres dans les échanges ioniques. Bien que l'on ait pu détecter la présence de radicaux libres après l'impact d'un faisceau sur du matériel biologique, ces effets sont difficiles à analyser, car ils sont souvent masqués par les effets thermiques ou photochimiques.

\section{IV.4. Les effets mécaniques}

Nous décrirons sous ce terme les effets obtenus à partir de phénomènes radicalement nouveaux, comme les effets multiphotoniques et non linéaires, qui prennent naissance lorsque l'on concentre sur de petites surfaces de matière le flux intense que produisent en de très brèves impulsions certains lasers déclenchés. Dans ce cas, un atome de la substance illuminée peut s'ioniser, c'est-à-dire absorber à la fois plusieurs photons du rayonnement laser et perdre un ou plusieurs de ses électrons. Dans le champ électrique intense de l'onde, les électrons s'accélèrent, bombardent les atomes, les ionisent par collision et engendrent, par un processus multiplicatif, un très grand nombre d'atomes ionisés qui forment un gaz ionisé ou plasma. C'est l'onde de choc qui accompagne la détente du plasma qui devient le moteur des effets mécaniques constatés, ceux-ci peuvent être assimilés à une micro-explosion. Ils entraînent une orientation de certaines molécules dans des directions privilégiées et, dans certains cas, peuvent repousser les cellules créant ainsi un véritable cratère.

Ces effets mécaniques ne s'observent qu'avec des impulsions inférieures à la microseconde. Ainsi, en ophtalmologie, le laser déclenché était initialement utilisé pour réaliser la plus petite lésion soudant la rétine à la choroïde en apportant le minimum d'énergie en un temps très court (30 ns). L'inconvénient à l'emploi de ce type de laser, est la vague de pression qui accompagne son action. Des pressions transitoires rencontrées au voisinage de la zone traitée furent la cause d'hémorragies ou d'autres séquelles. Cet inconvénient a disparu en employant un laser relaxé présentant des trains d'impulsions durant environ 1 milliseconde. Quand ils sont bien maîtrisés, ces effets mécaniques deviennent, au contraire, très utiles en ophtalmologie dans le traitement de certaines lésions comme 
les glaucomes, les ouvertures de membranule ou la section de brides vitréennes.

On voit donc que le mode d'action du laser sur la matière vivante est complexe et dépend non seulement du type d'appareil utilisé mais également de la façon dont se produit l'interaction entre le faisceau et le tissu biologique.

\section{LA NOTION DE CRITERE D'ATTEINTE ET DE SEUIL}

La détermination des niveaux d'exposition au-delà desquels une atteinte dommageable apparaît pose le problème de la nature des critères retenus, atteinte anatomique ou fonctionnelle, irréversible ou réversible, apparaissant dans des délais variables, rapides ou différés, et corrélativement de la sensibilité du test utilisé.

L'importance d'une lésion est également liée à la gêne fonctionnelle qu'elle provoque : une lésion irréversible, si elle se situe à la périphérie de la rétine, peut ne pas entraîner de dommage appréciable; fovéale, elle conduit généralement à une invalidité importante.

Par ordre croissant de sensibilité du test, il est classique de citer :

- l'examen ophtalmologique de la cornée, de la chambre antérieure, du cristallin et du fond d'œil associé ou non à l'étude et la mesure du champ visuel ;

- l'étude angiographique du fond d'œil ;

- l'examen anatomopathologique :

- en microscopie optique de la structure générale de la rétine,

- en microscopie électronique des ultrastructures, des photorécepteurs, des cellules ganglionnaires, des cellules de Müller.

Les examens fonctionnels et électrophysiologiques apportent des indications précieuses; ils mettent en évidence plus facilement les effets dommageables qui se traduisent par une atteinte fonctionnelle plus ou moins sévère. Mais il est important d'étudier l'acuité visuelle, le champ visuel, les courbes d'adaptation, le sens chromatique et l'activité électrophysiologique de la rétine et des aires visuelles de projection cérébrale. On sait, cependant, que les altérations électrophysiologiques sont essentiellement fonction de l'étendue de la surface rétinienne illuminée, du nombre de photorécepteurs atteints et de la localisation de cette atteinte sur la rétine. Leur étude est absolument nécessaire à la détermination des effets d'exposition de longue durée ou répétés, et ceci d'autant plus que l'illumination est diffusée et intéresse toute la rétine $[23,24,32,33]$.

La majeure partie des déterminations de seuil pour l'atteinte de la cornée, du cristallin ou de la rétine a été réalisée chez l'animal, le lapin et le primate macacca mulatta ou cynomolgus, plus rarement chez l'homme au cours de photocoagulation thérapeutique ou d'essais réalisés chez des sujets volontaires devant subir une énucléation. 
Les critères retenus sont presque toujours l'atteinte ophtalmoscopique ou l'observation photographique de la lésion, beaucoup plus rarement l'angiographie et l'histologie, exceptionnellement l'étude fonctionnelle.

II s'agit de lésions décelables 24 h après l'exposition, à l'exception de l'étude de l'apparition de cataracte plus tardive. Les illuminations sont réalisées dans des conditions variables et avec des diamètres d'image rétinienne différents: l'exposition comme l'énergie intraoculaire ou la densité surfacique d'énergie sont mesurées et/ou calculées à partir de la photométrie, dont la précision n'est pas toujours donnée. Les seuils sont exprimés à partir de résultats expérimentaux soumis à une analyse statistique utilisant le plus souvent la méthode des probits. L'ensemble des observations permet de dégager l'influence de trois facteurs principaux :

\section{V.1. La longueur d'onde}

Les UV-B et UV-C sont absorbés surtout par la cornée et la conjonctive provoquant photokératite et conjonctivite.

L'UV-A par effet photochimique provoque des lésions du cristallin et peut, pour des valeurs d'exposition en général plus élevées que l'UV-B et $\mathrm{C}$, provoquer des kératites par mécanisme photochimique.

La cornée non protégée comme la peau par le stratum cornéen est plus sensible aux UV. Alors que I'UV-A traverse plus facilement le cristallin, les effets caractéristiques des UV-B sont plus importants: l'efficacité est ici fonction de la longueur d'onde (à $350 \mathrm{~nm}, 50 \%$ de l'énergie parvient bien jusqu'au cristallin sans provoquer de lésion, alors que moins de $2 \%$ de l'énergie à $300 \mathrm{~nm}$ suffit à entraîner l'atteinte).

Dans l'UV-A, le visible et le proche IR-A, la rétine est concernée.

Dans I'IR-B et $C$, la transmittance oculaire diminue et les altérations les plus marquées sont celles de la cornée. Dans le visible, les travaux de HAM [16] sur le singe Rhésus (zone paramaculaire) montrent l'influence de différents facteurs et en particulier de la longueur d'onde (tableau II).

TABLEAU ॥

\begin{tabular}{|c|c|c|c|c|c|c|c|c|}
\hline Laser & $\stackrel{\lambda}{n m}$ & & $\mathrm{~s}$ & & $6 \mathrm{~s}$ & $100 \mathrm{~s}$ & $1000 \mathrm{~s}$ & Transmittance \\
\hline Nd. YAG & 1064 & 56.1 & \pm 5.3 & 37.5 & \pm 4.2 & $32.5 \pm 3.1$ & $24,0 \pm 3$ & 0.76 \\
\hline $\mathrm{He} . \mathrm{Ne}$ & 632.8 & 29.9 & \pm 1.4 & 15.2 & \pm 3 & $8,4 \pm 2,8$ & $5.4 \pm 2.3$ & 0.93 \\
\hline $\mathrm{Ar}$ & 610 & 22.0 & \pm 1.9 & 12.3 & \pm 1.1 & $8.1 \pm 1.1$ & $5.8 \pm 0.3$ & 0.92 \\
\hline $\begin{array}{l}\text { Laser à } \\
\text { colorant }\end{array}$ & 580 & 26.1 & \pm 4.5 & 11,5 & \pm 2.4 & $7.6 \pm 0.8$ & $4.0 \pm 1.7$ & 0,91 \\
\hline $\mathrm{Ar}$ & 514.5 & 14.5 & \pm 3.3 & 10,3 & \pm 2.3 & $2.2 \pm 0.5$ & $0,32 \pm 0,01$ & 0,87 \\
\hline $\mathrm{Ar}$ & 488 & 9.4 & \pm 1.2 & 6,1 & \pm 0.8 & $0,77 \pm 0,2$ & $0,15 \pm 0,8$ & 0.83 \\
\hline $\mathrm{Ar}$ & 457.8 & 5.1 & \pm 0.8 & 3,2 & $\pm 0,6$ & $0,52 \pm 0,1$ & $0,06 \pm 0,2$ & 0,69 \\
\hline $\mathrm{He} . \mathrm{Cd}$ & 441,6 & 0.91 & $\pm 0,3$ & 0,41 & $\pm 0,2$ & $0.20 \pm 0.2$ & $0,03 \pm 0,1$ & 0,45 \\
\hline
\end{tabular}

L'irradiance rétinienne est exprimèe en $\mathrm{W} . \mathrm{cm}^{-2}$ et l'image rétinienne est ici voisine de $500 \mathrm{um}$, le diamètre pupillaire est de $7 \mathrm{~mm}$. 
On retient dans le spectre visible que les seuils des brûlures rétiniennes sont, en général, beaucoup plus bas pour les émissions de faible longueur d'onde.

Dans l'ultraviolet, les seuils s'abaissent d'un facteur 60 environ pour les $\lambda$ de 230-250 à $300 \mathrm{~nm}$ comme le montrent les travaux de COGAN et al. [4], chez le lapin et le primate, et varient essentiellement selon la courbe d'absorption de la cornée et de la conjonctive, pour la longueur d'onde considérée.

L'étude de l'atteinte pour des lésions juste supra-liminaires révèle à énergie égale, des différences notables dans l'atteinte des différentes structures [19] ceci chez le lapin chinchilla et le primate, comme le montre le tableau III, exprimant schématiquement l'importance de la lésion en fonction de la longueur d'onde.

TABLEAU II

Importance de la lésion rétinienne en fonction de la longueur d'onde

\begin{tabular}{|c|c|c|c|c|c|}
\hline \multirow{2}{*}{$\begin{array}{c}\text { Laser krypton } \\
\text { et argon } \\
20 \mathrm{~mW} \cdot \mathrm{cm}^{-2} \text { (cornée) }\end{array}$} & \multicolumn{5}{|c|}{ Longueur d'onde (nm) } \\
\hline & $\begin{array}{l}674 \\
\text { rouge }\end{array}$ & $\begin{array}{l}568 \\
\text { jaune }\end{array}$ & $\begin{array}{l}530 \\
\text { vert }\end{array}$ & $\begin{array}{l}488 \\
\text { bleu }\end{array}$ & $\begin{array}{c}457 \\
\text { violet }\end{array}$ \\
\hline - Couche des fibres & + & + & \pm & \pm & \pm \\
\hline $\begin{array}{l}\text { - Couche des cellules gan- } \\
\text { glionnaires }\end{array}$ & + & & & & \\
\hline - Plexiforme interne & & & & & \\
\hline - Nucléaire interne & + & + & & + & + \\
\hline $\begin{array}{l}\text { - Nucléaire externe } \\
\text { (noyaux-cônes-bâtonnets) }\end{array}$ & +++ & ++ & ++ & +++ & ++ \\
\hline - Cônes et bâtonnets & + & & +++ & +++ & + \\
\hline - Epithélium pigmentaire & ++ & & ++ & + & + \\
\hline - Choroïde & ++ & & + & + & \\
\hline
\end{tabular}

Il existe en général :

- une atteinte plus importante du segment interne que du segment externe ;

- une zone d'absorption élective surtout pour les longueurs d'onde plus courtes à la jonction du segment interne et externe des photorécepteurs ;

- une accumulation de microcavitations dans la couche des photorécepteurs :

- une migration des mélanocytes le long des fibres de Müller ;

- une réponse plus marquée de l'épithélium pigmentaire et de la choroïde aux grandes longueurs d'onde ;

- une atteinte minime et égale en toute $\lambda$ de la couche nucléaire interne sans altération de la couche plexiforme interne ;

- une atteinte importante de la couche plexiforme externe et de la couche nucléaire externe ;

- une altération du tissu interstitiel rapide et majeure ;

- un œdème transitoire qui disparaît pour toute longueur d'onde en 4 à 6 jours. 
Si le seuil est 4 à 10 fois plus élevé, l'hémorragie apparaît immédiatement et des lésions plus complexes détruisent tout le tissu rétinien jusqu'à la sclère.

\section{V.2. La dimension de l'image rétinienne}

En général, l'irradiance rétinienne nécessaire pour provoquer une lésion est inversement proportionnelle au diamètre de l'image rétinienne $[14,15,22]$. On doit souligner que les impacts de petite dimension sont plus rapidement refroidis par la vascularisation que les impacts à surface large.

Chez le primate, dans le spectre visible, le seuil de l'irradiance rétinienne pour une image de $10 \mu \mathrm{m}$ à $1 \mathrm{~mm}$ varie de $1 \mathrm{~kW} . \mathrm{cm}^{-2}$ à environ $10 \mathrm{~W} . \mathrm{cm}^{-2}$.

Pour une impulsion de $\lambda=694,3 \mathrm{~nm}$ et de $30 \mathrm{~ms}$, le seuil varie, pour une image de $50 \mu \mathrm{m}$ à $1 \mathrm{~mm}$, de $1,5 \mathrm{J.cm}^{-2}$ à $0,02 \mathrm{J.cm}^{-2}$. A $514,5 \mathrm{~nm}$, pour une impulsion de $100 \mu$ et une image de 20 à $200 \mu \mathrm{m}$, l'irradiance rétinienne passe de $0,5 \mathrm{~J} \cdot \mathrm{cm}^{-2}$ à $0,15 \mathrm{~J} . \mathrm{cm}^{-2}$.

\section{V.3. Le temps}

Il semble que le modèle thermique soit, en général, bien adapté à la détermination des seuils de lésion lorsque la durée d'exposition est supérieure à $1 \mathrm{~ms}$; mais les résultats expérimentaux sont d'interprétation plus délicate et divergent lorsqu'il s'agit d'impulsions plus courtes.

Pour des expositions variant entre $10 \mu \mathrm{s}$ et $10 \mathrm{~s}$, le seuil augmente comme une fonction puissance $(3 / 4)$ de la durée t. Par contre, si la durée de l'impulsion est comprise entre $10 \mathrm{~ns}$ et $1 \mu \mathrm{s}$, la relation s'inverse. Pour les impulsions de l'ordre de la picoseconde, les résultats expérimentaux sont fort dispersés, mais les seuils en général augmentent.

En fait, les mécanismes sont peu connus, la photométrie comme la mesure du temps sont délicates et les résultats expérimentaux sont d'interprétation d'autant plus difficile que l'on veut déterminer l'exposition correspondant au seuil de lésion, non seulement d'impulsion unique mais de trains d'impulsions pendant des durées variables.

Les travaux de EBBERS et al. [5], LUND et al. [20], HEMSTREET et al. [17], SKEEN et al. [25], GIBBONS et al. [10], ont permis d'apporter des renseignements précieux dans le cas de laser à néodyme et à argon, dont la fréquence de répétition varie de 1 à $10 \mathrm{KHz}$ et de durée d'impulsion comprise entre 10 et $700 \mu$ d'une part, $10 \mu \mathrm{s}$ et $1 \mathrm{~ms}$ d'autre part, et pendant des durées d'exposition variant de 1 à $30 \mathrm{~s}$; mais les résultats expérimentaux sont, dans l'ensemble, trop fragmentaires pour permettre d'accorder une confiance totale aux facteurs de correction proposés dans les normes.

\section{COMPARAISON DE LA DETERMINATION EXPERIMENTALE D'UN SEUIL D'ATTEINTE IRREVERSIBLE DE LA RETINE AU MOYEN DE L'OPHTALMOSCOPIE SIMPLE ET APRES ANGIOGRAPHIE}

Le dispositif d'illumination est un laser pulsé à colorant initié par flash, dont la fréquence de récurrence maximale est de $15 \mathrm{~Hz}$ et dont la durée 
d'impulsion est de $600 \mathrm{~ns}$. Le spectre d'emission est dans le visible et correspond au spectre de fluorescence de la Rhodamine $G$.

Le faisceau est divisé en deux voies, l'une destinée à la photométrie à l'aide d'un détecteur pyroélectrique, l'autre illuminant l'œil de l'animal à la pupille dilatée par cycloplégique. Le système optique permet la formation sur la rétine de l'image d'un diaphragme circulaire de 2 à $4 \mathrm{~mm}$; selon la distance focale de l'animal adulte et de même âge, l'image rétinienne, chez le lapin, tout au moins, a un diamètre voisin de $285 \mu \mathrm{m}$ et $570 \mathrm{um}$. L'exposition est modifiée en interposant des filtres neutres de différente densité. Les mesures physiques sont connues avec une précision de $7 \%$.

La première étude concerne l'examen ophtalmoscopique du fond d'œil. Ces observations ont lieu $10 \mathrm{~min}, 1 \mathrm{~h}, 24 \mathrm{~h}, 8 \mathrm{j}, 15 \mathrm{j}$ après l'exposition. Afin d'éviter des expositions trop nombreuses et de modifier ainsi le seuil d'atteinte de la rétine, il n'est jamais délivré plus de trois impulsions et la plupart des observations sont limitées à une seule impulsion.

Les observations de la rétine ont été classées en 10 stades :

0 - Fond d'œil et transparence des milieux oculaires normaux ;

1 - Diminution de transparence des milieux oculaires;

2 - Opacité grise de la rétine (fig. 1) ;

3- Opacité rose ;

4 - Opacité blanche nacrée ;

5 - Opacité nacrée en cours de cicatrisation :

6 - Opacité nacrée entourée d'un halo rouge ;

7 - Opacité plus ou moins rouge entourée d'une ou plusieurs couronnes blanc nacré ;

8 - Opacité de stade 6 ou 7 en cours de cicatrisation ;

9 - Hémorragie rétinienne localisée ;

10 - Hémorragie rétinienne diffuse (fig. 2).

Le stade 1, fugace, qui dure quelques heures, est un phénomène de tyndallisation des milieux transparents de l'œil et surtout du vitré ; il empêche souvent de distinguer une lésion plus importante qui n'apparaîtra que 24 heures plus tard. II en résulte que la probabilité d'observer une lésion est maximale à $\mathrm{J}+1$.

Le stade 2, lésion minimale mise en évidence, constitue un stade de lésion irréversible. Elle s'accompagne toujours de lésion irréversible des photorécepteurs, mais la gêne fonctionnelle qui en résulte est fonction de sa localisation. Dans le cas du lapin, qui ne possède pas de fovea mais une zone allongée, la strie visuelle, comme dans le cas du singe au niveau de la macula, elle entraîne un scotome central important. II existe une réparation possible au stade 2 ; elle est suffisante pour faire disparaître la lésion à l'examen ophtalmoscopique si la lésion est suffisamment petite, mais si l'épithélium rétinien peut récupérer une pigmentation peu différente de la normale, l'organisation fonctionnelle des photorécepteurs et de la rétine n'est jamais restaurée au stade 2.

Pour trois longueurs d'onde et deux diamètres d'image rétinienne, l'expression du pourcentage d'atteinte en fonction de l'irradiance rétinienne ou de l'énergie intra-oculaire est donnée dans le tableau IV. 


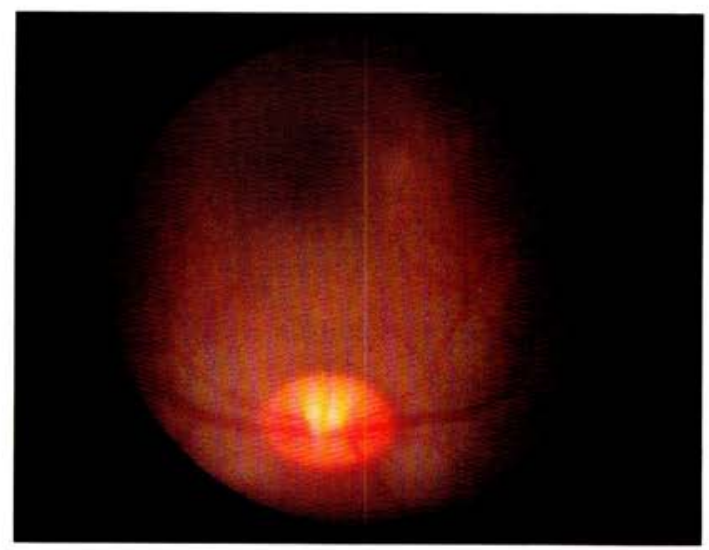

Fig. 1. - Lésion de stade 2 chez le singe, à J +1, $593 \mathrm{~mm}$, $600 \mathrm{~ns}$, irradiance rétinienne $0,24 \mathrm{~J} . \mathrm{cm}^{-2}$. La lésion est située sous la macula.

Fig. 2. - Lésion de stade 10 chez le singe, à JO; $593 \mathrm{~nm}$, $600 \mathrm{~ns}$, irradiance rétinienne $0,87 \mathrm{~J} . \mathrm{cm}^{-2}$
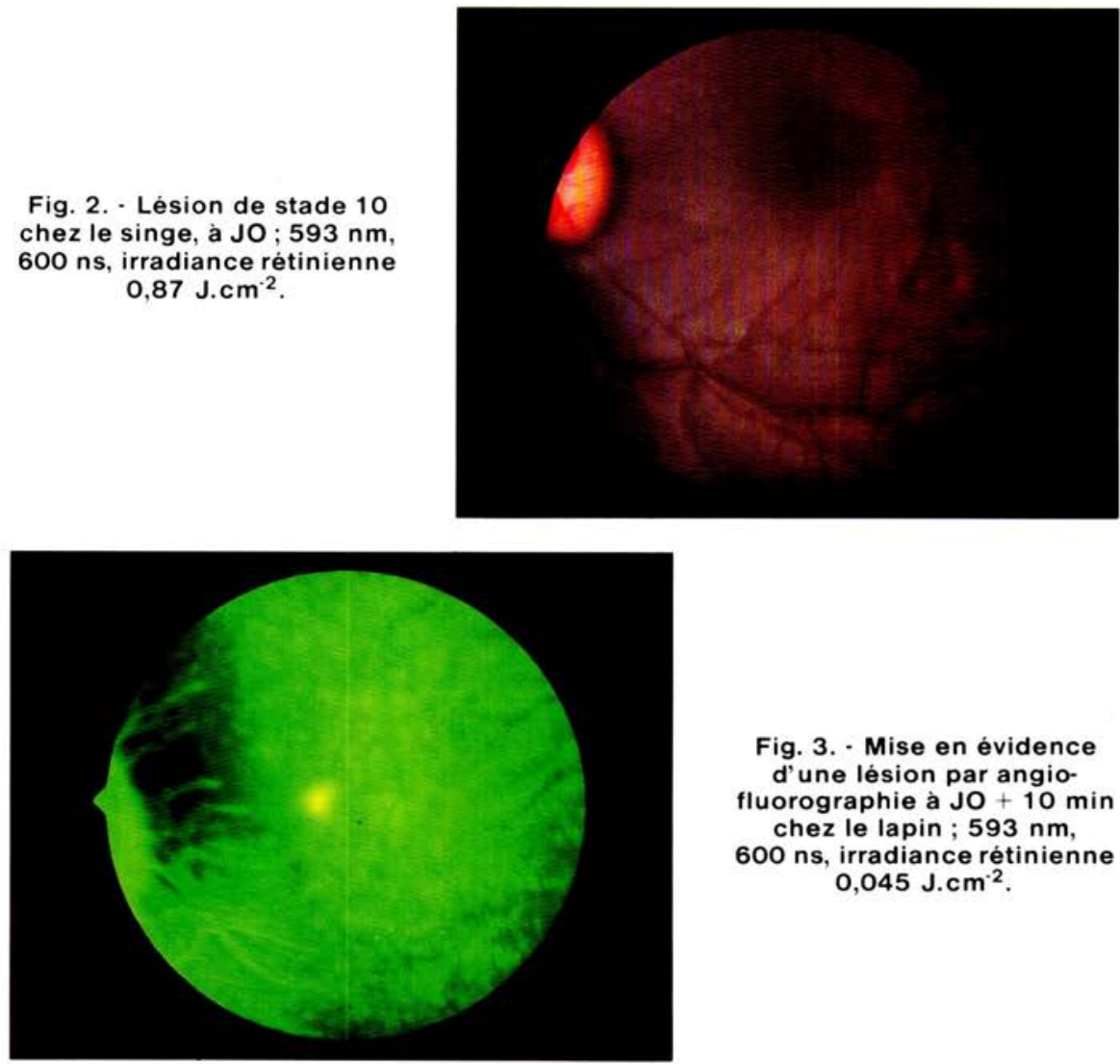

Fig. 3. - Mise en évidence d'une lésion par angiofluorographie à JO $+10 \mathrm{~min}$ chez le lapin ; $593 \mathrm{~nm}$, $600 \mathrm{~ns}$, irradiance rétinienne $0,045 \mathrm{~J} . \mathrm{cm}^{-2}$. 
TABLEAU IV

Atteinte de la rétine en fonction de l'irradiance rétinienne ou de l'énergie intra-oculaire

\begin{tabular}{|c|cc|cc|cc|cc|c|c|}
\hline $\begin{array}{c}\text { Pourcentage } \\
\text { d'atteinte }\end{array}$ & $\begin{array}{c}\lambda=610 \mathrm{~nm} \\
\mathrm{~S} 2\end{array}$ & \multicolumn{3}{|c|}{$\mathrm{s}$ S1 } & \multicolumn{2}{|c|}{$\mathrm{s}=593 \mathrm{~nm}$} & \multicolumn{4}{c|}{$\lambda=580 \mathrm{~nm}$} \\
\hline 1 & 0,4 & 1,12 & 11,7 & 8 & 3,8 & 10 & 6,2 & 4,3 & 9,1 & 25 \\
10 & 7,6 & 21 & 110 & 76 & 59 & 162 & 15,3 & 10 & 119 & 327 \\
30 & 92 & 251 & 322 & 221 & 219 & 601 & 280 & 192 & 406 & 1114 \\
50 & 290 & 797 & 530 & 364 & 403 & 1105 & 1083 & 743 & 718 & 1970 \\
90 & 1098 & 3013 & 940 & 645 & 813 & 2231 & 5125 & 3516 & 1384 & 3799 \\
100 & 1393 & 3824 & 1042 & 715 & 922 & 2532 & 6771 & 4645 & & \\
\hline
\end{tabular}

$\mathrm{S} 1=$ Surface rétinienne $=0,000638 \mathrm{~cm}^{2}\left(D_{1}=0,285 \mathrm{~mm}\right), \mathrm{S} 2=$ Surface rétinienne $=$ $0,002552 \mathrm{~cm}^{2}\left(\mathrm{D}_{2}=0,570 \mathrm{~mm}\right)$.

Le premier chiffre exprime l'irradiance rétinienne en $\mathrm{mJ} . \mathrm{cm}^{-2}$; Le deuxième chiffre donne l'énergie intra-oculaire absorbée exprimée en $\mu \mathrm{J}$.

L'atteinte est l'observation à $\mathrm{J}+1$ du stade 2 , plus petite lésion irréversible décelable. La répartition du domaine énergétique explorée de 0,001 à $1,5{\mathrm{~J} . \mathrm{cm}^{-2}}^{2}$ a été réalisée selon une loi logarithmique en 9 classes. L'ajustement de la relation dose-effet a été réalisé avec une fonction de la forme : $\log _{n} D=\log _{n} B+K \log _{n} X$

où

$D$ est l'énergie délivrée

B est le cœfficient

$X$ est le pourcentage de sujets atteints.

Le seuil de probabilité retenu est $p=0,005$.

Dans une deuxième série expérimentale sur un lot de 100 sujets, subissant une ou deux expositions, l'application de la méthode angiographiqúe à la fluorescéine a permis d'établir de nouvelles relations doseeffet. L'angiographie à la fluorescéine consiste à injecter une solution à $10 \%$ de fluorescéine dans le système circulatoire et à observer et photographier le fond d'œil éclairé en lumière bleue, dans une bande de longueur d'onde excitant électivement la fluorescéine (fig. 3).

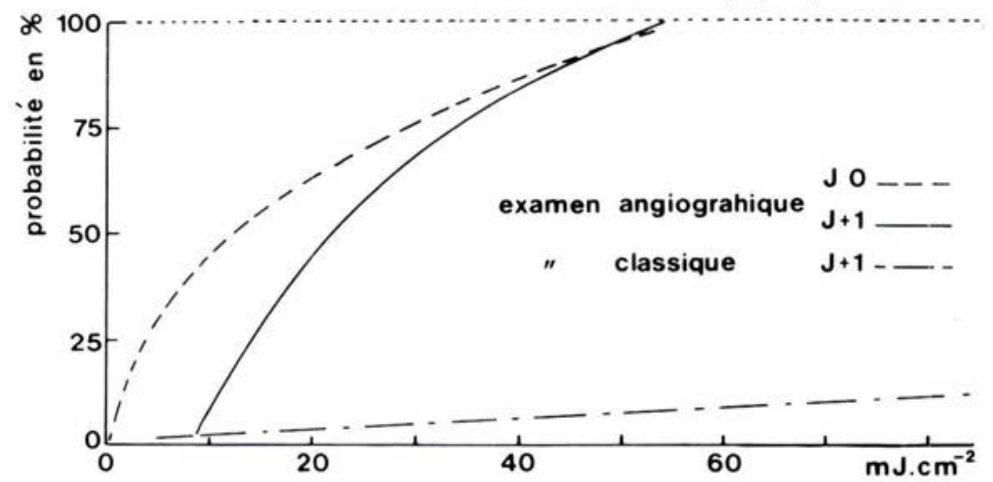

Fig. 4 - Probabilité d'observer chez le lapin une lésion rétinienne en fonction de l'énergie délivrée au niveau de la rétine. 
Les résultats sont rassemblés dans les tableaux $V$ et $\mathrm{VI}$ et la figure 4 , exprimant le pourcentage de lésions observées en fonction de l'irradiance.

TABLEAU V

Irradiance rétinienne en $\mathrm{mJ} . \mathrm{cm}^{-2}$

\begin{tabular}{|c|c|c|c|}
\hline \multirow{3}{*}{$\begin{array}{l}\text { Pourcentage } \\
\text { d'atteinte }\end{array}$} & \multirow{2}{*}{$\frac{\lambda=593 \mathrm{~nm}}{\substack{\text { Angiofluorographie } \\
\text { à } \mathrm{H}+1}}$} & \multicolumn{2}{|c|}{ Surface rétinienne $0,00255 \mathrm{~cm}^{2}$} \\
\hline & & $\begin{array}{l}\text { Angiofluorographie } \\
\qquad a \mathrm{~J}+1\end{array}$ & $\begin{array}{l}\text { Ophtalmoscopie simple } \\
\text { à } J+1\end{array}$ \\
\hline & & & \\
\hline 1 & 0,0014 & 9,8 & 3,77 \\
\hline 5 & 0,056 & 10,5 & 25,76 \\
\hline 10 & 0,276 & 11,4 & 58,94 \\
\hline 20 & 1,30 & 14,6 & 134,86 \\
\hline 50 & 11,27 & 32,2 & 402,83 \\
\hline 75 & 28,65 & 33,5 & 653,77 \\
\hline 100 & 55,60 & 50,8 & 922,80 \\
\hline Relation & & & \\
\hline dose/effet & $D=B \cdot X^{m}$ & $D=B+m \log _{n} X$ & $\mathrm{D}=\mathrm{B} \cdot \mathrm{X}^{\mathrm{m}}$ \\
\hline B & 11,2598 & $-129,04225$ & 0,14197 \\
\hline $\mathrm{m}$ & 0,43579 & 56,3556 & 0,8367 \\
\hline$\pi$ & 0,99446 & 0,998 & 0,91 \\
\hline$t$ & 18,92 & 27,34503 & 4,907 \\
\hline$p$ & 0,001 & 0.001 & 0,005 \\
\hline $\mathrm{t}$ & 8.61 & 12.94 & 4,773 \\
\hline
\end{tabular}

TABLEAU VI

Energie intra-oculaire exprimée en $i \mathrm{~J}$

\begin{tabular}{|c|c|c|c|}
\hline \multirow{2}{*}{\begin{tabular}{c} 
Pourcentage \\
\cline { 2 - 4 } d'atteinte
\end{tabular}} & $\begin{array}{c}\text { Angiofluorographie } \\
\text { a J0 }\end{array}$ & $\begin{array}{c}\text { Surface rétinienne }=0.00255 \mathrm{~cm}^{2} \\
\text { Angiofluorographie } \\
\text { à J }+1\end{array}$ & $\begin{array}{c}\text { Ophtalmoscopie simple } \\
\text { à J }+1\end{array}$ \\
1 & & & \\
5 & 0,004 & 27,7 & 10,3 \\
10 & 0,15 & 29,8 & 70,5 \\
20 & 0,76 & 32,4 & 162 \\
50 & 3,6 & 41,5 & 370 \\
75 & 30,9 & 63 & 1105 \\
100 & 78,6 & 95 & 1794 \\
\hline
\end{tabular}

Limite d'exposition : $5,10^{-7} \mathrm{~J} . \mathrm{cm}^{-2}$ à la cornée, c'est-à-dire pour une pupille de $7 \mathrm{~mm}$ : $0,19 \mu \mathrm{J}$ intra-oculaire (l'image rétinienne est alors ponctuelle). 
On observe que, sur cette même série expérimentale, l'irradiance nécessaire pour observer un œdème de la rétine décelable $1 \mathrm{~h}$ après l'exposition est beaucoup plus faible que celle correspondant à l'observation de la lésion stade 2 . A $\mathrm{J}+1$, une irradiance de $0,060 \mathrm{~J}^{-\mathrm{cm}^{-2}}$ suffit à provoquer l'apparition d'un œdème du fond de l'œil avec une probabilité de $100 \%$; l'énergie nécessaire pour observer en ophtalmoscopie simple un stade 2 avec la même probabilité est, dans les mêmes conditions expérimentales, de $0,960 \mathrm{J.cm}^{-2}$. Les études en cours sur Macacca mulatta et sur Macacca cynomolgus mettent en évidence le même phénomène. Le fait qu'à probabilité égale l'irradiance requise pour observer une tache fluorescente sur la rétine est beaucoup plus faible que celle nécessaire pour provoquer l'apparition d'une dépigmentation stade 2 pourrait laisser supposer que le phénomène œdème ne s'accompagne pas de lésion définitive.

Or, l'étude histologique d'une lésion décelable par angiographie

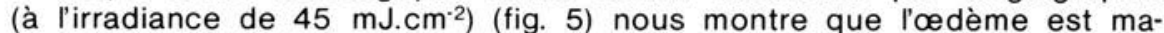
nifeste au niveau de la couche des photorécepteurs, une séparation et une dissociation apparaissant nettement entre les segments externes et les segments internes. La lésion ne touche essentiellement que l'épi-

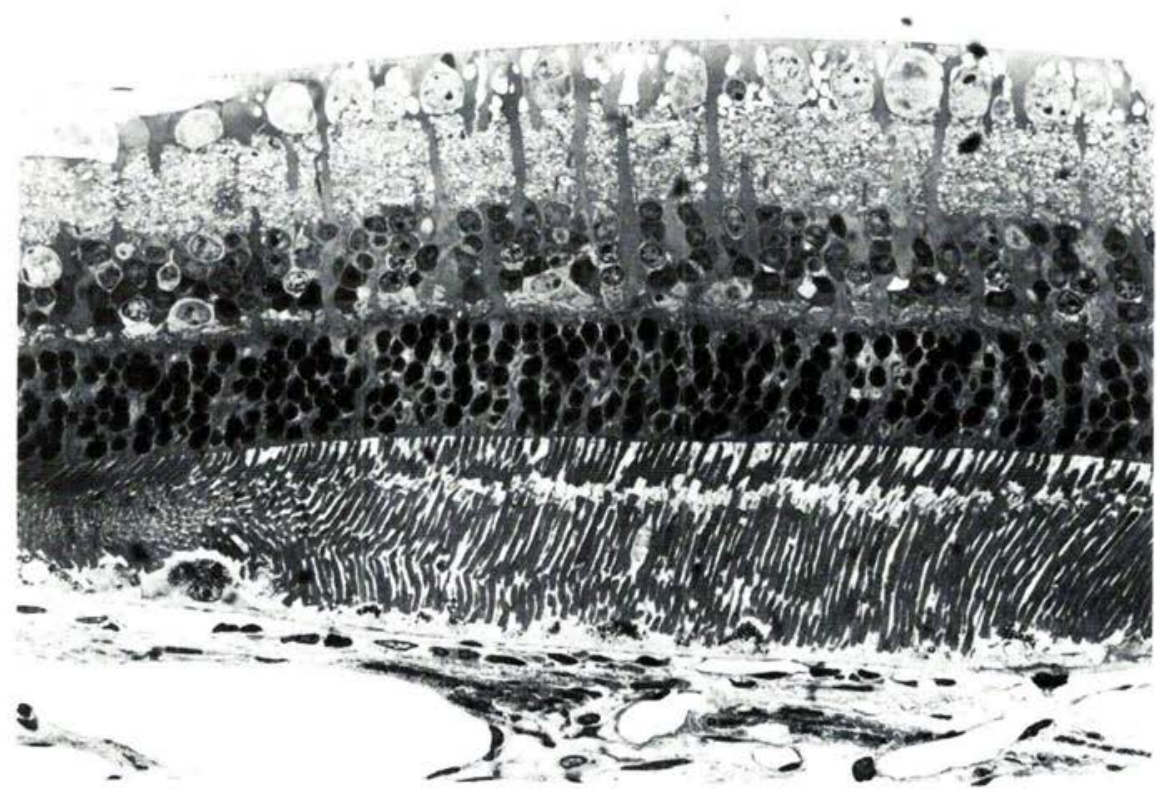

Fig. 5 - Partie centrale d'une lésion observée $24 \mathrm{~h}$ après une impulsion laser délivrant $45 \mathrm{~mJ} . \mathrm{cm}^{-2}$ à la rétine. 
thélium pigmentaire, la couche des photorécepteurs et les prolongements des cellules de Müller, situés sur la limitante interne mais elle est définitive. A la différence des irradiances plus fortes (115 à $150 \mathrm{~mJ}^{-\mathrm{cm}^{-2}}$ ) les couches nucléaires externe et interne ne sont pas lésées par cette irradiation et on n'observe pas de noyaux pycnotiques ou de vacuolisation dans ces deux couches.

L'exposé de ces nombreux paramètres comme l'analyse de ces résultats conduisent à plusieurs réflexions: l'établissement des normes de sécurité est réalisé à partir d'une compilation des travaux expérimentaux (tableau VII) et il est généralement fait appel aux conditions les plus défavorables: adaptation nocturne et ouverture pupillaire maximale de $7 \mathrm{~mm}$. Partant de l'observation d'un effet irréversible et de l'exposition permettant d'observer $50 \%$ de sujets lésés, un facteur de sécurité $F$ variant entre 10 et 100 est appliqué et la valeur limite d'exposition est définie par l'expression :

$$
V L E=D L 50 \% \times \frac{1}{F}
$$

II apparaît alors immédiatement que l'évaluation des normes est essentiellement fonction :

- du critère de l'atteinte, de sa nature et de la date de l'observation par rapport à l'illumination,

- de la relation dose-effet et surtout du pourcentage d'atteinte choisi : $1,10,33,50$ ou $100 \%$,

- de l'incertitude de certaines données expérimentales et de la complexité des phénomènes étudiés.

On sait que pratiquement seule l'étude des lésions irréversibles de la rétine, de la cornée ou du cristallin préside à l'établissement des recommandations actuelles. Si le fait qu'elles ont été déterminées sur l'animal ne constitue pas un obstacle majeur [7], il apparaît nécessaire de préciser les effets d'illumination répétée ou de longue durée, de déterminer de façon précise le délai acceptable entre deux expositions à peine infra liminaires, de connaître l'effet d'impulsions ultra-courtes, d'approfondir la nature des mécanismes des échanges énergétiques dans les illuminations brèves et répétées et de généraliser les études d'effets fonctionnels. II est nécessaire de s'attacher non pas tant au critère anatomique qu'aux atteintes fonctionnelles se traduisant par une baisse de la sensibilité rétinienne, une modification plus ou moins irréversible de l'adaptation, une altération plus ou moins définitive du sens chromatique. Sachant que les phénomènes étudiés ne sont pas stochastiques au sens propre du terme, il nous paraît préférable d'évaluer les limites d'exposition admissibles à partir d'une probabilité d'atteinte de $10 \%$, et dans tous les cas de préciser la valeur de cœfficients de sécurité en faisant référence à la nature des critères utilisés. Si l'on admet, enfin, qu'il est nécessaire de réaliser un effort de présentation des normes actuellement diffusées, il sera, ces réserves faites, plus facile d'en faire admettre la validité. 
EFFETS DU FAISCEAU LASER SUR LA FONCTION VISUELLE

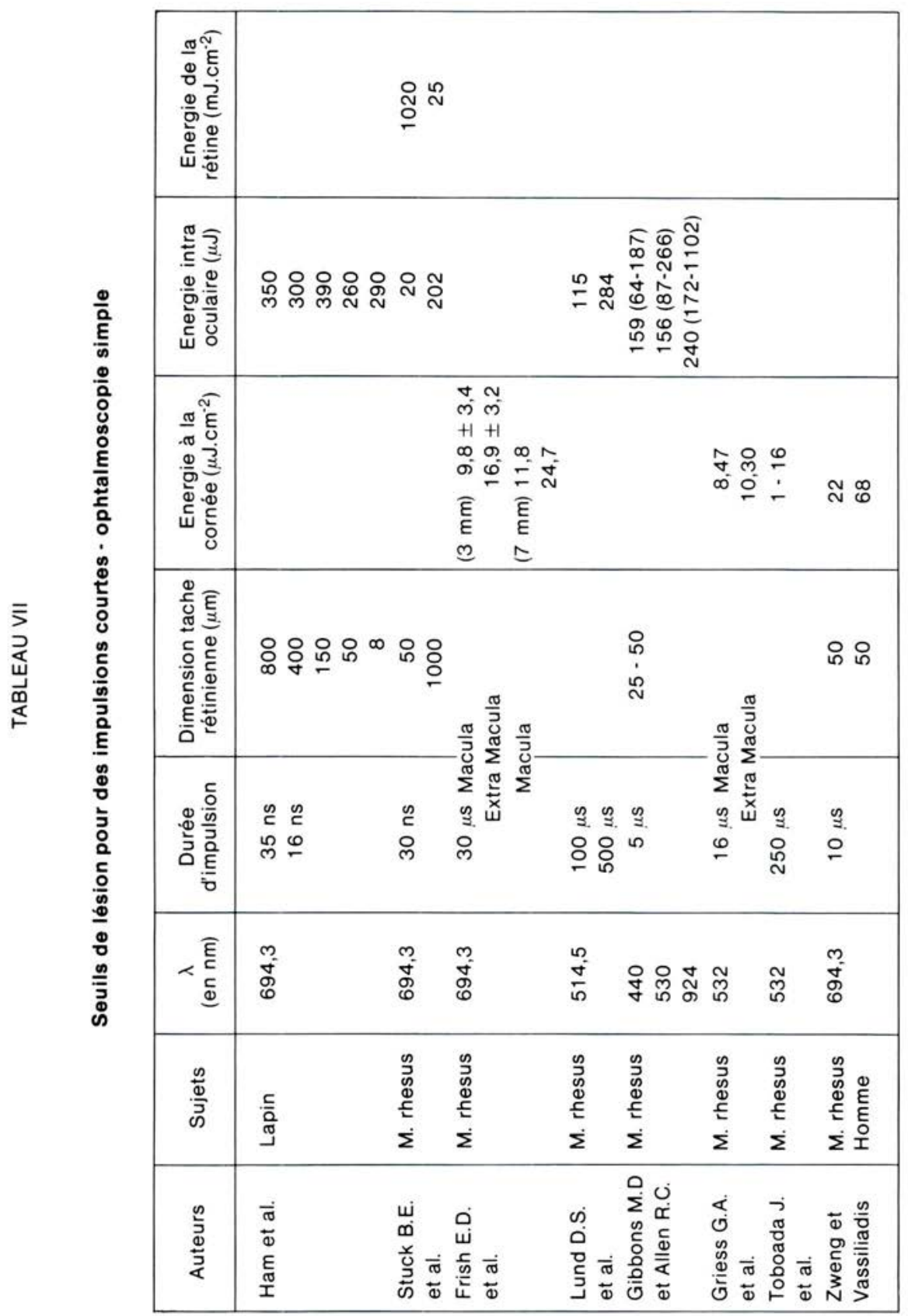




\section{BIBLIOGRAPHIE}

[1] BEATRICE E.S. and FRISH G.D. Retinal laser damage thresholds as a function of image diameter. Arch. Environ. Health, 1973, 27, 322-326.

[2] BOETTNER, E.A. and WOLTER J.R. Transmission of the ocular media. Invest. Ophtal. 1962, 1, 776-783.

[3] BOETTNER E.A. Spectral transmission on the eye, final report AD-663 246, 1967.

[4] COGAN D.G. and KINSEY W.E. Action spectrum of keratitis produced by UV radiation. Arch. Opht., 1967, 77, 670-677.

[5] EBBERS R.W. and DUNSKY I.L. Retinal damage threshold for multiple pulse lasers. Aerospace Med., 1973, 44, 317-318.

[6] FRISH G.D., BEATRICE E.S. and HOLSEN C.R. Comparative study of argon and ruby retinal damage thresholds. Invest. Ophtalmol., 1971, 10, 911-919.

[7] GABEL V.P. and BIRGRUBBER R. A comparative study of threshold laser lesions in the retinae of human volunteers and rabbits. Health Phys., 1980, 40, 238-240.

[8] GEEREATS W.J. and BERRY E.R. Ocular spectral characteristics as related to hazards from lasers and other light sources. Am. J. Ophtalm., 1968, 66, 15-20.

[9] GIBBONS W.D. and ALLEN R.E. Retinal damage from supra threshold Q-switched laser exposure. Health Phys., 1978, 35, 461-469.

[10] GIBBONS W.D. and EGBERT D.E. Ocular damage thresholds for repetitive pulse laser exposures. USAF Technical Report SAM-TR-74, Brooks Air Force Base, Tx : School of Aerospace Medicine, 1979 (AD-777 144/7).

[11] GRIESS A.G., BLANKENSTEIN M.F. and WILLIFORD C.G. Ocular damage from multiple pulses laser exposure. Health Phys., 1980, 39, 912-917.

[12] GUBISCH R.W. Optical performance of the human eye. J. Opt. Soc. Am, 1967, $57,407-415$.

[13] HAM W.R. Jr., WILLIAMS R.C., MUELLER H.H., RUFFIN R.S., SCHMIDT F.H., CLARKE A.M., VOS J.J. and GEERAETS W.J. Ocular effects of laser radiation. Acta Ophtal., 1965, 390-409.

[14] HAM W.T. Jr., GEERAETS W.H., WILLIAMS R.C., GUERRY D. and MUELLER H.A. Laser radiation protection. In : 1. International congress of radiation protection. Pergamon Press, New York : 1968, 933-943.

[15] HAM W.T. Jr., MUELLER H.A., WILLIAMS R.C. and GEERAETS W.J. Ocular hazard from viewing the sun unprotected and through various windows and filters. Appl. Opt., 1973, 12, 2122-2129.

[16] HAM V.T., MUELLER H.A., RUFFOLO J.J. and CLARKE A.M. Sensitivity of retina to radiation damage as a function of wavelength. Photochem. Photobiol., 1978, 29, 735-743.

[17] HEMSTREET H.W., BRUCE W.R., ALTOBELLI K.K., STEVENS C.C. and CONNOLLY J.S. Ocular hazards of picosecond and repetitive pulsed lasers. Second annual report, 1974-1975. USAF Contract, San Antonio, Tx: Technology Incorporated, 1975.

[18] L'ESPERANCE F.A. Jr. The ocular histopathologic effect of krypton and argon laser radiation. Am. J. Opht., 1969, 68, 263-273.

[20] LUND D.J., CARVER C. and ZWICKER B. Ocular damage threshold study. Frankford Arsenal Memorandum, Report M70-24-1. Philadelphia : Frankford Arsenal, 1973.

[21] LUND D.J. and BEATRICE E.S. Ocular hazard of short pulse argon laser irradiation. Lair Report $n^{\circ} 42,1977$. 
[22] MARSHALL J. Thermal and mechanical mechanisms in laser damage to the retina. Invest. Opht., 1970, 9, 97-115.

[23] ROBBINS D.O., ZWICK H. and HOLST G.C. A method for producing foveal retineal exposures in a awake, task-oriented rhesus monkey. Behav. Res. Meth. Instrum., 1973, 5, 457-461.

[24] ROBBINS D.O., ZWICK N. and HOLST G. Functionnal assessment of laser exposures in awake task-oriented rhesus monkey. Mod. Probl. Ophtalmol., $1974,13,284-290$.

[25] ROULIER A. Calculation of temperature increase in the eye produced by intense light. Bull. Math. Biophys., 1970, 32, 403.

[26] SKEEN G.H., BRUCE W.R., TIPS J.H., SMITH M.C. and GARSA G.C. Ocular effect of repetitive laser pulses, final report. San Antonio, Tx: Technology Inc. 1972 (AD 746 795).

[27] STUCK B.E. Comparaison of the maximum permissible exposure for Q-switched ruby laser exposures. Letterman Army Institute of Research, Presidio of San Francisco, California 94129, Laboratory Report n 35, 1976.

[28] TOBOADA J. and GIBBONS W.D. Retinal tissue damage induced by single ultra short laser light pulses. Appl. Optics, 1978, 17, 2871-2873.

[29] VOS J.J. Heat-damage to the retina by lasers and photocoagulator, Ophthalmologia $1966,151,652-654$.

[30] WRAY J.L. Model for prediction of retinal burns, Technical safety study, Washington D.C., DASA, 1962 (DASA-1282).

[31] ZWENG H.C., VASSILIADIS A., PEPPERS N.A. and PEABODY R.R. Thresholds of laser eye hazards. 33th Annual Meeting Industrial Hygiene Fundation, Oct. 15, 1968.

[32] ZWICK H., BEDELL R.B. and BLOOM K. Spectral and visual deficits associated with laser irradiation. Mod. Probl. Ophtalmol., 1974, 299-306.

[33] ZWICK H. Visual functionnal effects ol laser irradiation. Association for research in vision and Ophtalmology, Spring, 1974.

\section{BIBLIOGRAPHIE GENERALE ET PRINCIPALES RECOMMANDATIONS}

- ETATS-UNIS. Army Environmental Hygiene Agency, Laser hazards bibliography, Aberdeen Proving Ground, MD 21010, 1980.

- AMERICAN NATIONAL STANDARDS INSTITUTE. Safe use of lasers. ANSI Standard 2-136, 1, New York: ANSI, 1980.

- AMERICAN CONFERENCE OF GOVERNMENTAL INDUSTRIAL HYGIENISTS (ACGIH), Guide for Control of laser Hazards, 1976. Thresholds limits values. Cincinnati : ACGIH, 1981.

- LASER INSTITUTE OF AMERICA. Laser Safety Guide. Cincinnati : L.I.A., 1981.

- ETATS-UNIS. Department of labor, occupational safety and health administration, Code of Federal Regulations Title 29, Part 1926, 54, Non lonizing radiation. 1978.

- ETATS-UNIS. Department of the air force. Laser health control. AFM 161-8, 1980.

- ETATS-UNIS. Department of the army. Control of hazards to health from laser radiation, TB-MED-279, 1975.

- ETATS-UNIS. Department of health, education and welfare. Code of Federal Regulations Title 21, Part 1040, Performance standards for light-emitting products, 1978. 
- FRANCE. Bulletin officiel de la direction des personnels civils de la Défense Nationale. BOC/PP-France, 1975, $n^{\circ} 7,359-364$.

- CLUET A. et MAYER H. Risques liés à l'utilisation industrielle des lasers. Cahier Notes Doc., 1980, n 99, 207-222.

- INTERNATIONAL ELECTROTECHNICAL COMMISSION (I.E.C.). Technical Commitee on laser products. Genève : IEC (en préparation).

- BRITISH STANDARDS INSTITUTION. Protection of personel against hazards from laser radiation, B.S. 4083, 1972, 1981 (soumis pour approbation).

- AGARD. Laser hazards and safety in the military environnement. L.S. - 79, 1975.

- ORgANISATION MONDIALE DE LA SANTE (OMS) Lasers and optical radiation. (Environmental Health Criteria, 23) Genève : OMS 1982.

- J. HAUT, S. LIMON, M. MASSIN, G. PERDRIEL. Lasers en opthalmologie. Paris : Masson, 1981.

- COURT. L., CheVAleRAud P. et SANTUCCI G. Effets biologiques des lasers. In: "Effets biologiques des rayonnements non ionisants: utilisation et risques associés". 19. Congrès international de Nainville-les-Roches, 22-26 mai 1978. S.I. : SFRP, 1978. 366-431. 